\title{
Accuracy of 64-Multidetector-Row Computed Tomography in the Diagnosis of Coronary Artery Disease
}

\author{
Mehraj Sheikh ${ }^{a} \quad$ AbdelMohsen Ben-Nakhic A. Mohemad Shukkur ${ }^{d}$ \\ Tariq Sinan $^{\mathrm{a}}$ Ibrahim Al-Rashdan ${ }^{\mathrm{b}}$ \\ Departments of a Radiology and ${ }^{\mathrm{b}}$ Medicine, Cardiology Division, Faculty of Medicine, Kuwait University, \\ 'Department of Radiology, Mubarak al Kabeer Hospital, and d Department of Medicine, Cardiology Division, \\ Chest Hospital, Ministry of Health, Kuwait
}

\section{Key Words}

64-slice multidetector computed tomography coronary angiography $\cdot$ Coronary artery disease $\cdot$ Coronary angiography

\begin{abstract}
Objectives: The aim of this prospective study was to assess the accuracy of 64-multidetector-row computed tomography coronary angiography (CTA) in the diagnosis of coronary artery disease (CAD). Patients and Methods: Ninety-two patients suspected of having CAD underwent CTA using a 64slice CT scanner before a scheduled, conventional coronary angiogram (CCA). Blinded assessment of CTA to detect CAD was performed. The accuracy of CTA in detecting significant stenoses ( $\geq 50 \%)$ was compared to CCA. Data analysis was performed on 73 patients because the scans were nondiagnostic in 5 patients and 14 refused to undergo coronary angiography. Results: The CTAs of 21 of these 73 patients were considered as normal; 19 were confirmed on CCA. For the remaining 52 diagnosed as abnormal, 51 were confirmed on CCA. For patient-based analysis, CTA had a sensitivity of $95 \%$, a specificity of $96 \%$, a positive predictive value of $98 \%$ and a negative predictive value of $90 \%$. For the whole vessel, the
\end{abstract}

sensitivity of CTA was $60-100 \%$, for all vessels and the specificity was $82-100 \%$. Pooled sensitivity was $92 \%$ and pooled specificity was $98 \%$. For the segments, the sensitivity of CTA was $64 \%$ or above for all vessels except for the distal left anterior descending artery (40\%), mid circumflex artery (50\%) and posterior descending artery (60\%); the pooled sensitivity was $79 \%$. The specificity for the segments was $82-100 \%$ for all vessels and pooled specificity was $94 \%$. Conclusion: The sensitivity and specificity for patient-based analysis and for the main coronary vessels were high whereas for the segments, the sensitivity was moderately good, but the specificity was high, confirming that a negative CTA is useful to rule out significant CAD. A coordinated classification system between radiologists and cardiologists is required to eliminate errors in segment classification.

Copyright $\odot 2009$ S. Karger AG, Basel

\section{Introduction}

Globally, coronary artery disease (CAD) continues to be one of the leading causes of morbidity and mortality. Conventional invasive catheter coronary angiography (CCA) currently remains the gold standard in the diag-

\section{KARGER \\ Fax +41613061234 E-Mail karger@karger.ch} www.karger.com
(C) 2009 S. Karger AG, Basel

$1011-7571 / 09 / 0184-0323 \$ 26.00 / 0$

Accessible online at: www.karger.com/mpp
Dr. Mehraj Sheikh

Department of Radiology, Faculty of Medicine

Kuwait University, PO Box 24923

13110 Safat (Kuwait)

E-Mail mehraj@hsc.edu.kw 
nosis of CAD. In the UK, about $25 \%$ of coronary angiograms show normal coronary arteries [1] while in the USA, nearly $70 \%$ of the patients undergo invasive angiography for suspected disease [2]. In Kuwait, the figures are not known, but the burden of CAD is high [3]. Hence a reliable and reproducible noninvasive diagnostic test for the diagnosis and grading of coronary stenosis is highly desirable to avoid unnecessary invasive testing. Multidetector row computed tomography is the most promising method at present. Compared to single-detector-row CT, which was introduced in 1981, 4-detector-row CT developed in 1998 was 8 times more performing than singledetector-row CT, but the results were not consistent [4] and therefore this method was not put into clinical use. By 2002, further improvements in technology led to the emergence of 16-row-detector CT coronary angiography (CTA) which was reported to be clinically useful [4]. Now, with the emergence of 64-detector CT scanner, the improvement in spatial and temporal resolution and sophisticated ECG gating allow fast and detailed cardiac imaging that appears to be nearly as accurate as the traditional invasive diagnostic technique [5-8]. This study was undertaken using a 64-row-detector CT scanner to evaluate the sensitivity and specificity of CTA in identifying significant CAD using invasive CCA as the gold standard.

\section{Patients and Methods}

\section{Patients}

Patients from the Kuwait Heart Center who were referred for CCA were given the option of having CTA before the performance of a scheduled CCA. Patients who gave consent were referred to the Department of Radiology, Mubarak Al Kabeer Hospital for CTA examination prior to CCA. Exclusion criteria for CTA were atrial fibrillation, high baseline heart rate ( $>70$ beats/ min) with contraindication to $\beta$-blockade, known allergic reaction to iodinated contrast agents, renal insufficiency, severe chronic congestive heart failure and any previous percutaneous coronary intervention or coronary artery bypass graft. Patients with heart rates more than 70 beats/min were prescribed 50 $100 \mathrm{mg}$ oral metoprolol to keep the heart rate below 60 beats/min. Nitrates were not used before the procedure. The study was approved by our Ethics Review Committee.

\section{Scanning Protocol and Image Reconstruction}

All patients were scanned with a 64-slice CT scanner (LightSpeed VCT, GE, Milwaukee, Wisc., USA). The angiographic scanning parameters used are shown in table 1 . A bolus of 100-120 ml contrast medium (Ultravist-300) followed by 50-60 $\mathrm{ml}$ of normal saline was injected through an arm vein at a flow rate of $4-5 \mathrm{ml} / \mathrm{s}$ using a dual injector. Twenty milliliters of contrast medium was used during the bolus timing scan calculated at the level of the
Table 1. Angiographic scan parameters

$\begin{array}{ll}\text { Number of slices per rotation } & 64 \times 1 \\ \text { Individual detector width } & 0.625 \mathrm{~mm} \\ \text { Rotation time } & 0.35 \mathrm{~s} \\ \text { Pitch } & 0.20: 1 \text { to } 0.325: 1 \\ & \text { (based on the patient's heart } \\ & \text { rate and rotation speed) } \\ \text { Tube voltage } & 120 \mathrm{kVp} \\ \text { Tube current } & 320 \mathrm{~mA}\end{array}$

ascending aorta using MIROI software. Five to eight seconds were added to the calculated MIROI time to perform the coronary angiogram study. All data sets acquired were reconstructed from the axial images using retrospective electrocardiogram gating with the GE Advantage Windows Workstation 4.2. The data set was reconstructed at $75 \%$ of R-R intervals. In 7 cases with insufficient image quality due to motion artifacts, additional data sets were reconstructed between 25 and $85 \%$ of R-R interval and images of optimal quality were selected.

\section{CTA and CCA Image Evaluation}

The interventional radiologists (M.S. and A.M.) evaluated the reconstructed images independently using visual estimation of coronary artery narrowing. Axial images (fig. 1a) were reviewed in addition to postprocessing volume-rendered 3D images (fig. 1b), and maximum-intensity projections (fig. 1c), multiplanar reconstructions (fig. 1d), and linear conformation (fig. 1e) of the vessels were used to assess vessel stenosis. An aggregate judgment was made after viewing the various images and the best images that delineated the stenosis were selected. The CTA reporters were asked to determine whether the coronary artery or segments were assessable on the CTA scan, and for assessable coronary segments, the presence of significant stenosis (defined as $\geq 50 \%$ reduction in lumen diameter) was determined (fig. 2a, b). Coronary artery anatomy was divided into 13 segments according to a modification of the classification of the American Heart Association (table 2). Any disagreement between the two CTA reporters on a particular coronary segment was resolved by consensus. An interventional cardiologist blinded to the results of CTA findings performed the CCA within 1 month after the CTA examination and visually evaluated the images for degree of stenosis. A significant lesion was defined as $50 \%$ or more reduction in lumen diameter. Comparisons were then made between CTA and CCA findings. The results were evaluated based on patient outcome (presence or absence of CAD), main coronary vessels involved, i.e. left main artery (LMA); left anterior descending (LAD); circumflex artery (Cx); first diagonal; second diagonal; obtuse marginal; right coronary artery (RCA); and the posterior descending artery (PDA), and finally by the segments involved (proximal, mid and distal segments of major vessels, terminal small-caliber vessels and side branches) as shown in table 2.

Ninety-two patients underwent CTA. Five subjects were considered nondiagnostic, due to motion artifacts related to high heart rate ( $>70$ beats $/ \mathrm{min}$ ) in 4 , and due to poor contrast opacification of the vessels in 1 . The remaining 87 CTA scans were con- 
Fig. 1. a CTA showing axial image. b 3Dvolume-rendered image of the left coronary system. c Maximum-intensity projection image of coronary artery. $\mathbf{d}$ Curved multiplanar image of the right coronary artery. e Linear conformation of the right coronary artery.
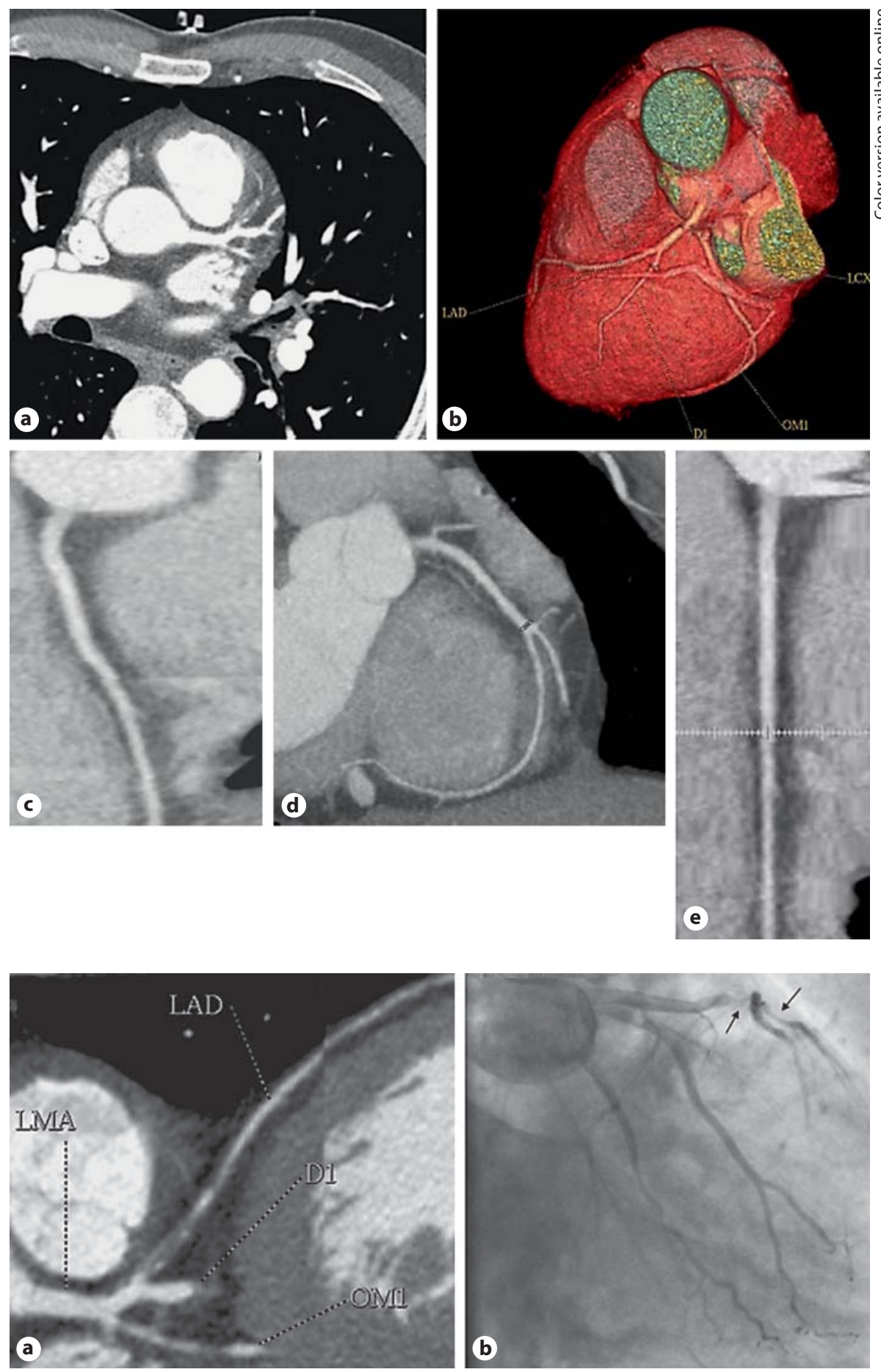

Fig. 2. a Maximum-intensity projection image showing diseased LAD segment. b Confirmed on CCA (arrows).

sidered diagnostic, but 14 patients refused to undergo CCA. Final analyses were performed in 73 (60 males, 13 females) patients.

\section{Statistical Analysis}

Each artery was summarized by a pair of statistical parameters that measured CT accuracy. CCA was used as the gold standard.
These pairs of summary statistical parameters included sensitivity (proportion of positive CT scans among patients with positive angiography) and specificity (proportion of negative CT scans among patients with negative angiography). The confidence intervals of sensitivity and specificity were calculated using the $\mathrm{F}$ distribution method to compute the exact confidence limits for 
Table 2. Coronary vessels and their segment

\begin{tabular}{ll}
\hline Vessel & Segments \\
\hline Right coronary artery & 1 = proximal, $2=$ mid, $3=$ distal \\
Posterior descending artery & 4 \\
Left main artery & 5 \\
Left anterior descending & $6=$ proximal, $7=$ mid, $8=$ distal \\
First diagonal & 9 \\
Second diagonal & 10 \\
Circumflex & $11=$ proximal, $12=$ mid \\
Obtuse marginal & 13 \\
\hline
\end{tabular}

Table 3. Patient characteristics $(n=73)$

\begin{tabular}{lcc}
\hline & Number & Percent \\
\hline Male & 60 & 82.2 \\
Age, years & $60 \pm 9(32-67)$ & - \\
Allergies & 4 & 5.5 \\
Diabetes mellitus & 38 & 52.1 \\
Hypertension & 39 & 53.4 \\
Hyperlipidemia & 65 & 89.0 \\
Smoking & 37 & 50.7 \\
Peripheral vascular disease & 3 & 4.1 \\
Heart rate, beats/min & & \\
$\quad<60$ & 54 & 73.97 \\
$\quad 60-69$ & 13 & 17.8 \\
$\quad 70-79$ & 6 & 8.2 \\
\hline
\end{tabular}

Table 4. Sensitivity and specificity of various coronary arteries at $95 \% \mathrm{CI}$

\begin{tabular}{lcr}
\hline Blood vessel & Sensitivity, \% & \multicolumn{1}{c}{ Specificity, \% } \\
\hline Left main artery & $100(0.48-1.0)$ & $94(0.86-9.8)$ \\
Left anterior descending & $100(0.89-1.0)$ & $90(0.76-0.97)$ \\
First diagonal & $89(0.67-0.99)$ & $100(0.93-1.0)$ \\
Second diagonal & $100(0.03-1.0)$ & $100(0.95-1.0)$ \\
Circumflex & $84(0.60-0.97)$ & $98(0.90-1.0)$ \\
Obtuse marginal & $87(0.60-0.98)$ & $100(0.94-1.0)$ \\
Posterior descending artery & $60(0.15-0.95)$ & $97(0.90-1.0)$ \\
Right coronary artery & $94(0.80-0.99)$ & $100(0.91-1.0)$
\end{tabular}

Pooled sensitivity $92 \%$ and pooled specificity $98 \%$. Figures in parentheses are confidence intervals.
Table 5. Sensitivity and specificity of various coronary vessel segments at $95 \%$ CI

\begin{tabular}{lcc}
\hline Blood vessel & Sensitivity, \% & Specificity, \% \\
\hline LMA & $100(0.48-1.0)$ & $94(0.86-9.8)$ \\
Proximal LAD & $64(0.35-0.87)$ & $90(0.79-0.96)$ \\
Mid LAD & $92(0.73-0.99)$ & $82(0.68-0.91)$ \\
Distal LAD & $40(0.12-0.74)$ & $87(0.77-0.94)$ \\
D1 & $89(0.67-0.99)$ & $100(0.93-1.0)$ \\
D2 & $100(0.03-1.0)$ & $100(0.95-1.0)$ \\
Proximal Cx & $82(0.48-0.98)$ & $95(0.87-0.99)$ \\
OM & $87(0.60-0.98)$ & $100(0.94-1.0)$ \\
Mid Cx & $50(0.21-0.79)$ & $100(0.94-1.0)$ \\
PDA & $60(0.15-0.95)$ & $97(0.90-1.0)$ \\
Proximal RCA & $88(0.62-0.98)$ & $91(0.81-0.97)$ \\
Mid RCA & $80(0.56-0.94)$ & $89(0.77-0.96)$ \\
Distal RCA & $90(0.55-1.0)$ & $92(0.82-0.97)$ \\
\end{tabular}

LMA = Left main artery; LAD = left anterior descending; $\mathrm{D} 1$ = first diagonal; $\mathrm{D} 2$ = second diagonal; $\mathrm{Cx}=$ circumflex; $\mathrm{OM}=$ obtuse marginal; $\mathrm{PDA}=$ posterior descending artery; $\mathrm{RCA}=$ right coronary artery.

the binomial proportion $(\mathrm{x} / \mathrm{n})$ and the results for each artery were then combined to get an overall summary statistic. The sensitivity and specificity were pooled as the sum of true positive divided by the sum of diseased and the sum of true negative divided by the sum of nondiseased, respectively. The software used for statistical analysis was Meta-Disc, version 1.4, Universidad Complutense, Madrid, Spain.

\section{Results}

\section{Patient-Based Analysis}

The characteristics of the 73 patients analyzed are summarized in table 3 . Of these, 21 were considered as normal based on CTA; 19 of these were confirmed on CCA. For the remaining 52 subjects diagnosed as abnormal on CTA, 51 were confirmed by CCA while only 1 subject proved to be normal. Hence, for the patient-based analysis, CTA had a sensitivity of $95 \%$ and a specificity of $96 \%$, a positive predictive value of $98 \%$ and a negative predictive value of $90 \%$ when compared to CCA.

\section{Vessel-Based Analysis}

For the 8 vessels (table 2), the sensitivity of CTA ranged from $60 \%$ (for PDA) to $100 \%$ (for LMA) and the pooled sensitivity was $92 \%$. The specificity of CTA ranged from 82 to $100 \%$ for all vessels and the pooled specificity was 98\% (table 4). 


\section{Segment-Based Analysis}

For the 13 segments (table 2), the sensitivity of CTA was $\geq 64 \%$ for all vessels except for the distal LAD (40\%), mid $\mathrm{Cx}(50 \%)$ and PDA (60\%). Pooled sensitivity was $79 \%$. The specificity of CTA was $\geq 82 \%$ for all vessels and the pooled specificity was $94 \%$. The sensitivity of individual segments of LAD, RCA, and the $\mathrm{Cx}$ artery ranged from 40 to $92 \%, 80$ to $90 \%$ and 50 to $82 \%$, respectively, while the sensitivity for the whole vessel was 100, 94 and $84 \%$, respectively (table 5). Hence, the sensitivity of CTA for some segments (distal LAD, mid Cx and PDA) was less compared to the whole vessel.

\section{Discussion}

Progress has been accomplished in the management of patients with suspected CAD in the previous two decades. Traditionally, these patients were hospitalized and required 2-3 days of hospital stay to complete a diagnostic evaluation. Subsequently, coronary-care observation units were established that provided accelerated diagnostic protocols for such patients that decreased the length of hospital stays to approximately half a day, decreased the costs, and resulted in fewer missed myocardial infarctions, with comparable clinical outcomes. The advent of multidetector CTA, especially with 64-slice CT scanners, has taken this evolution a step further with a technology that appears to be as accurate as CCA even in the assessment of distal coronary artery branches [5-7].

Using 16-slice CT, overall sensitivity including all segments was reported to be between 73 and 95\%, depending on the diameter of the segment, the modality of analysis, and patient selection criteria [7,9-11]. Sensitivity is shown to be higher when only larger vessels are assessed and the smaller vessels are excluded from analysis [10-13].

The 64-slice CT scanner, because of its superior spatial and temporal resolution, has the ability to assess smallercaliber vessels (less than 1.5-2.0 mm) and therefore demonstrates significant improvement in the assessment of coronary artery branches. In contrast to the 16-slice CT scanner, no significant difference in sensitivity has been reported with the 64-slice scanner when comparing proximal with middle or distal coronary artery segments [6]. A sensitivity of up to $99 \%$ and a specificity of up to 95\% compared with invasive CCA have been reported with 64 -slice CTA $[5,6,14]$ which was confirmed by our study, where both sensitivity and specificity were high (sensitivity 95 and 92\%, specificity 96 and 98\%, respec- tively) in patient-based and vessel-based analysis alike. High sensitivity and specificity with 64-slice scanners have been shown even in patients with coronary calcification, tachycardia and obesity [15].

However, at the segment level, the sensitivity (79\%) was moderately good whereas the specificity (94\%) also was high in contrast to the results of Leschka et al. [6], who reported both high sensitivity and high specificity at the segment level in their study. The pooled sensitivity for the vessel-based analysis improved from 79 to $92 \%$ after excluding the small segments from the analysis. In fact, for the main vascular tree (RCA, LAD and the Cx arteries) the sensitivity improved up to 100,94 and $84 \%$, respectively. Similar observations of improved sensitivity and specificity after excluding the small vessels have been made by others $[11,12]$. The spatial resolution of CTA is lower $\left(0.4^{3}\right)$ than that of the CCA $\left(0.2^{3}\right)$; therefore, the interpretation of small distal segments by CTA may be limited, and stenoses of such segments can be missed or overestimated. In our study, since some stenoses of proximal larger-sized segments were misdiagnosed, the discordance between CTA and CCA in locating disease in the vessel segments most likely suggests divergences between the radiologists and the cardiologists. Consensus on segmental demarcation may decrease the degree of divergences in terms of classification that currently exist. At the segment level, the specificity also was high in our study, but the sensitivity was only moderate.

Motion artifacts and heavy coronary calcification are the major limitations to reliable assessment of all coronary artery segments [7, 9]. An optimal heart rate less than 60 beats/min to reduce motion artifacts is achieved in most patients taking oral $\beta$-blockers (50-100 mg daily) prior to the $\mathrm{CT}$ examination or with intravenous metoprolol (5-15 mg) in patients with insufficient chronotropic response to the oral dose. Despite improved temporal resolution in 64-slice scanners, $\beta$-blockers are required in patients with heart rates more than 70 beats/ min in order to obtain acceptable images. In addition, it is possible to compensate for motion artifacts in the majority of affected vessels by individually choosing the phase with minimal vessel motion for each segment. We routinely gave only oral $\beta$-blockers to our patients; in 4 cases, the desired heart rate was not achieved, and the images in these cases were suboptimal. A dual-source CT could be an alternative method in patients with higher heart rates $[16,17]$. 


\section{Conclusion}

Our study revealed high specificity and sensitivity for both the patient-based and the vessel-based analysis whereas at the segment level the sensitivity was only moderate, but the specificity again was high, confirming that a negative CTA is useful to rule out significant CAD. To eliminate discordance of segment classification, a coordinated classification system between the radiologists and the cardiologists is required.

\section{Acknowledgements}

This research work was supported by Kuwait University Research Grant No. MT 01/06. We would like to thank Mr. Nobel Abraham for his contribution in running the Advantage Windows software program.

\section{References}

1 Papaconstantinou HD, Marshall AJ, Burrell CJ: Diagnostic cardiac catheterisation in a hospital without on-site cardiac surgery. Heart 1999;81:465-469.

$\checkmark 2$ Sones FM Jr, Shirey EK: Cine coronary arteriography. Mod Concepts Cardiovasc Dis 1962:31:735-738.

3 Zubaid M, Rahsed W, Husain M, Mohammad BA, Ridha M, Basharuthulla M, Abu Rezq M, Smid J, Thalib L: A registry of acute myocardial infarction in Kuwait: patient characteristics and practice patterns. Can J Cardiol 2004;20:783-787.

-4 Manghat NE, Morgan-Hughes GJ, Marshall AJ: Multi-detector row computed tomography: imaging the coronary arteries. Clin Radiol 2005;60:939-952.

5 Mollet NR, Cademartiri F, van Mieghem CA, Runza G, McFadden EP, Baks T, Serruys PW, Krestin GP, de Feyter PJ: High-resolution spiral computed tomography coronary angiography in patients referred for diagnostic conventional coronary angiography. Circulation 2005;112:2318-2323.

-6 Leschka S, Alkadhi H, Plass A, Desbiolles L, Grünenfelder J, Marincek B, Wildermuth S: Accuracy of MSCT coronary angiography with 64-slice technology: first experience. Eur Heart J 2005;26:1482-1487.
Ropers D, Baum U, Pohle K, Anders K, Ulzheimer S, Ohnesorge B, Schlundt C, Bautz W, Daniel WG, Achenbach S: Detection of coronary artery stenoses with thin-slice multidetector row spiral computed tomography and multiplanar reconstruction. Circulation 2003; 107:664-666.

8 Musto C, Simon P, Nicol E, Tanigawa J, Davies SW, Oldershaw PJ, Arcuri N, Fox K, Di Mario C: 64-Multislice computed tomography in consecutive patients with suspected or proven coronary artery disease: initial single center experience. Int J Cardiol 2007; 114:90-97.

-9 Martuscelli E, Romagnoli A, D’Eliseo A, Razzini C, Tomassini M, Sperandio M, Simonetti G, Romeo F: Accuracy of thin-slice computed tomography in the detection of coronary stenoses. Eur Heart J 2004;25: 1043-1048.

10 Mollet NR, Cademartiri F, Nieman K, Saia F, Lemos PA, McFadden EP, Pattynama PM, Serruys PW, Krestin GP, de Feyter PJ: Multislice spiral computed tomography coronary angiography in patients with stable angina pectoris. J Am Coll Cardiol 2004;43: 2265-2270.

11 Nieman K, Cademartiri F, Lemos PA, Raaijmakers R, Pattynama PM, de Feyter PJ: Reliable non-invasive coronary angiography with fast submillimeter multislice spiral computed tomography. Circulation 2002; 106:2051-2054.

12 Soon KH, Chaitowitz I, Cox N, MacGregor L, Eccleston D, Bell KW, Kelly AM, Lim YL: Diagnostic accuracy of 16-slice CT coronary angiography in the evaluation of coronary artery disease. Australas Radiol 2007;51: 365-369.
3 Duncan RC, Wilde P, Oberhoff M, Rogers CA, Karsch KR, Baumbach A: Multislice computed tomography coronary angiography in patients admitted with a suspected acute coronary syndrome. Int J Cardiovasc Imag 2007;23:603-614

14 Tan KT, Reed D, Howe J, Challenor V, Gibson M, McGann G: CT vs. conventional angiography in unselected patients with suspected coronary heart disease. Int J Cardiol 2006;121:125-126.

15 Raff GL, Gallagher MJ, O’Neill WW, Goldstein JA: Diagnostic accuracy of noninvasive coronary angiography using 64-slice spiral computed tomography. J Am Coll Cardiol 2005;46:552-557.

16 Flohr TG, McCollough CH, Bruder H, Petersilka M, Gruber K, Süss C, Grasruck M, Stierstorfer K, Krauss B, Raupach R, Primak AN, Küttner A, Achenbach S, Becker C, Kopp A, Ohnesorge BM: First performance evaluation of a dual-source CT (DSCT) system. Eur Radiol 2006;16:256-268.

17 Achenbach S, Ropers D, Kuettner A, Flohr T, Ohnesorge B, Bruder $\mathrm{H}$, Theessen $\mathrm{H}$, Karakaya M, Daniel WG, Bautz W, Kalender WA, Anders K: Contrast-enhanced coronary artery visualization by dual-source computed tomography - initial experience. Eur J Radiol 2006;57:331-335. 